\title{
MULTILINEAR MIXING OPERATORS AND LIPSCHITZ MIXING OPERATOR IDEALS
}

\author{
Dahmane Achour, Elhadj Dahia and M. A. S. Saleh
}

Abstract. In [15], E. A. Sánchez Pérez introduced the class of $(s ; q, \theta)$-mixing operators, as a generalization of $(s ; q)$-mixing operators. We investigate analogous concepts here for the case of multilinear operators between Banach spaces and Lipschitz mappings between metric spaces, introducing the class of $\left(s, q ; p_{1}, \ldots, p_{m} ; \theta\right)$-mixing multilinear operators and the Lipschitz Banach ideal of $(s, q, \theta)$-mixing mappings show that our approach provides a multilinear and Lipschitz extension of quotient theorem like the linear case. Several characterizations of these mappings are presented, especially, every Lipschitz $(s, q)$-mixing map is Lipschitz $(s, q, \theta)$-mixing map and a result relies on the duality theory for $(q, \theta)$-absolutely Lipschitz operators are given.

Mathematics subject classification (2010): Primary 47B10, Secondary 47L22, 46A32.

Keywords and phrases: Lipschitz operators, Lipschitz $(s, q)$-mixing map, $(s, \theta)$-absolutely Lipschitz mappings, $(s ; q, \theta)$-mixing operators, mixing multilinear operators, quotient theorem.

\section{REFERENCES}

[1] D. Achour, E. Dahia, P. Rueda And E. A. SÁnchez PÉREZ, Factorization of strongly $(p, \sigma)$ continuous multilinear operators, Linear and Multilinear Algebra 62, No. 12 (2014) 1649-1670.

[2] D. Achour, P. Rueda And R. Yahi, $(p, \sigma)$-absolutely Lipschitz operators, Ann. Funct. Anal. 8 (1) (2017), 38-50.

[3] D. Achour, P. Rueda, E. A. SÁnchez PÉRez And R. YAhid, Lipschitz operator ideals and the approximation property, J. Math. Anal. Appl. 436 (2016), 217-236.

[4] R. F. AREns And J. Eels JR., On embedding uniform and topological spaces, Pacific J. Math 6 (1956), 397-403.

[5] J. A. Chávez-Domínguez, Lipschitz ( $q, p)$-mixing operators, Proc. Amer. Math. Soc. 140 (2012), 3101-3115.

[6] J. A. ChÁvez-DomíngueZ, Duality for Lipschitz p-summing operators, J. Funct. Anal. 261 (2011), 387-407, Lipschitz (q,p) -mixing operators, Proc. Amer. Math. Soc. 140 (2012), 3101-3115.

[7] E. Dahia, On the tensorial representation of multi-linear ideals, $\mathrm{Ph}$. D. thesis, University of Mohamed Boudiaf, M'sila (Algeria), 2014.

[8] E. Dahia, D. Achour And E. A. SAncheZ-Perez, Absolutely continuous multilinear operators, J. Math. Anal. Appl. 397 (2013) 205-224.

[9] H. JARCHOW, U. MATTER, Interpolative constructions for operator ideals, Note. Mat. (1) 8 (1988), 45-56.

[10] J. A. Lopez Molina, And E. A. SÁnChez PÉREZ, Ideales de operadores absolutamente continuos, Rev. Real Acad. Ciencias Exactas, Pisicas Y Naturales, Madrid 87 (1993), 349-378.

[11] U. MATTER, Absolute continuous operators and super-reflexivity, Math. Nachr 130 (1987), $193-216$.

[12] A. Pietsch, Operator Ideals, Deutsch, Verlag Wiss, Berlin, 1978; North-Holland, AmsterdamLondon-New York-Tokyo, 1980.

[13] M. A. S. SALEH, Nonlinear operator ideals (part I), arXiv:1507.00861 [math AF].

[14] M. A. S. SALEH, New types of Lipschitz summing maps between metric spaces, Math. Nachr 290 (2017), 1347-1373.

[15] E. A. SÁnChez PÉREZ, Products and quotients of $(p, \sigma)$-absolutely continuous operator ideals, Tr. J. of Mathematics 20 (1996) 323-334. 
[16] E. A. SÁNCHEZ PÉREZ, Ideals de operadores absolutamente continuos y normas tensoriales asociadas, PhD Thesis, Universidad politécnica de Valencia, Spain, 1997.

[17] I. Sawashima, Methods of Lipschitz duals, Lecture Notes Ec. Math. Sust. 419, Springer Verlag (1975), pp. 247-259.

[18] C. A. S. SoARes, Aplicacoes multilineares e polinômios misto somantes, PhD Thesis, Universidade Estadual de Campinas (UNICAMP), 1998.

[19] N. Weaver, Lipschitz Algebras, World Scientific Publishing Co. Singapore (1999). 